\title{
CAMPONESES E APICULTURA: UM ESTUDO DE CASO NO MACIC̣O COLOMBIANO
}

\author{
Diana Carolina Cadena Bastidas ${ }^{2}$ \\ Vanilde Souza Esquerdo ${ }^{3}$
}

\begin{abstract}
Resumen
Los sistemas productivos desarrollados por comunidades campesinas se caracterizan por una amplia red de relaciones sociales, que se entrelazan por el saber en la práctica. Por tanto, el objetivo de este artículo fue analizar el proceso de surgimiento y establecimiento de la actividad apícola en un grupo de campesinos del Sur-Occidente colombiano, agrupados en la Asociación de Apicultores del Macizo Colombiano- APIMACIZO. La metodología aplicada fue historia oral, usando como recurso metodológico relatos orales. Dentro de los resultados se evidencia la articulación de diferentes sectores a la práctica apícola, así como, la intervención de algunos sectores estatales en el proceso de formación de la Asociación. Las narraciones proporcionadas por algunos de los miembros de APIMACIZO permitió entender la ligación de diferentes sectores (iglesia, familia, vecinos) en la vida del campesinado de esta Asociación con la práctica apícola; donde la articulación se dio por medio del saber. Es a partir del saber en la apicultura que se consolidaron los lazos de amistad, vecindad, compadrazgo y las relaciones familiares.
\end{abstract}

Palabras clave:

Campesinado. Relaciones familiares. Historia Oral. Memoria Colectiva.

\footnotetext{
Abstract

The productive systems developed by peasant communities, are characterized by a wide network of social relationships, which are intertwined by practical knowledge. Therefore, the objective of this article was to analyze the process of emergence and establishment of beekeeping activity in a group

${ }^{1}$ Este artículo es una versión modificada de la propuesta inicial de trabajo presentado en la VIII Jornada de Estudos em Assentamentos Rurais, en junio de 2017.

${ }^{2}$ Doutoranda em Engenharia Agrícola na área de concentração em Gestão de Sistemas na Agricultura e Desenvolvimento Rural na Universidade Estadual de Campinas, Campinas - SP, Brasil. E-mail: kdnita20@gmail.com

${ }^{3}$ Doutora em Engenharia Agrícola (Unicamp). Professora da Universidade Estadual de Campinas, Campinas - SP, Brasil. Email: vanilde.esquerdo@feagri.unicamp.br
} 
of farmers from south-western Colombia, grouped in the Association of Beekeepers of the Colombian Massif - APIMACIZO. The applied methodology was oral history, using oral accounts as a methodological resource. Within the results it is evident the connection of different sectors to the beekeeping practice, as well as the intervention of some official sectors in the formation process of the Association. The accounts provided by some of the members of APIMACIZO made it possible to understand the coordination between different sectors (church, family, neighbors) in the life of the peasantry of this association with the apiculture practice; the coordination was possible by means of knowledge. It is from knowledge in beekeeping that bonds of friendship, neighborhood, deep friendship and family relationships were consolidated.

Keywords: Peasantry. Family relationships. Oral history. Collective memory.

\section{Introducción}

El mundo rural ha sufrido significativas transformaciones. La imagen que por mucho tiempo permaneció sobre el campesino como un simple productor, no corresponde a la dinámica de los procesos sociales del cual él es partícipe; al contrario, afirmamos que debe ser reconocido como una forma social de producción, en el sentido de ser responsable por la subsistencia de su familia, la cual es fundamento en la forma de organización en el trabajo, en el cual predominan espacios naturales, relaciones de proximidad y de interconocimiento (WANDERLEY, 2010; 2014).

Para el caso de Colombia y según el Programa de las Naciones Unidas para el Desarrollo PNUD (2011), "las tres cuartas partes de los municipios del país son predominantemente rurales (75,5\%)" (PNUD, 2011, p.18), asegurando que "esa ruralidad más amplia, compleja y no reconocida desborda los marcos institucionales actuales [...] deja ver con claridad que hay más ruralidad que institucionalidad pública; en pocas palabras, más ruralidad que Estado" (Ibíd.).

Al respecto, Forero et al. (2013) afirma que "la permanencia y reproducción de las comunidades rurales ha quedado demostrada con su persistencia en medio de las condiciones tan extremadamente difíciles y hostiles que han tenido que enfrentar en el campo colombiano" (FORERO et al., 2013, p.73). Es este campesinado que se ha mantenido en el tiempo, y aunque no ha estado exento de las dinámicas propias del mundo rural. Según Fals Borda (2002) a pesar del impacto de la modernidad capitalista en la agricultura, la clásica vocación campesina para producir alimentos y relacionarse con el medio ambiente no ha desaparecido.

$\mathrm{Y}$ si el campesinado no se ha extinto, las relaciones tierra, trabajo, familia y vecindad tampoco. Así lo evidencian algunos datos del Censo Nacional Agropecuario Colombiano (CNA) del año 2014 , los cuales afirman que el $22,8 \%$ de los residentes en la unidad productiva agropecuaria (UPA) declaran que su producción es para el autoconsumo, mientras que el 3,6\% tienen como finalidad el intercambio o trueque (DANE, 2014). Partiendo de que, la relación tierra, trabajo y campesino van mas allá de una forma mercantil, el presente artículo tiene como objetivo analizar el proceso de surgimiento y establecimiento de la actividad apícola en un grupo de campesinos del SurOccidente colombiano, actualmente agrupados en la Asociación de Apicultores del Macizo Colombiano- APIMACIZO, en el municipio de La Vega, Cauca.

Como intentaremos exponer, esta Asociación es el resultado de un proceso histórico en donde convergen varios actores tanto locales como externos a la comunidad, lo cual no solo ha permitido un desarrollo económico del grupo de campesinos allí involucrados, sino que se ha establecido un vínculo entre una actividad productiva específica - apicultura- y la identidad del campesinado.

Por lo tanto, a partir de la implementación de la metodología historia oral, la discusión será abordada en tres partes: en el primer apartado, Surgimiento y establecimiento de la apicultura en la región, discutiremos la entrada de la apicultura en la región a partir de la memoria oral de los miembros de la Asociación, por lo que será analizada la memoria colectiva, donde es traída en el sentido de Halbwachs (2004), para quien se puede hablar de memoria colectiva, cuando evocamos un hecho, el cual ocupa un lugar en la vida de nuestro grupo y que hemos expuesto en el momento en que lo recordamos, desde el punto de vista del grupo. Sin embargo, dentro de la memoria también hay saberes, creencias, emociones que son transmitidas y hacen parte de las prácticas culturales de un grupo (JELIN, 2002). De allí que Brandão (1986) y Brandão (1994) aporta teóricamente la conexión entre saber e identidad. Porque si bien, es a partir de la memoria colectiva que se hace alusión al papel de la iglesia en el proceso de aprendizaje de esta "nueva" práctica productiva en 
cabeza de uno de sus representantes, a través del saber transmitido se logran evidenciar los lazos sociales que se van estableciendo durante la práctica apícola. De esta manera, para analizar el establecimiento de la práctica apícola, optaremos por mantener el orden cronológico y jerárquico de las memorias orales, en donde prima un proceso de difusión entre amigos, vecinos y familiares en manos de un poblador de la región.

El segundo apartado, La localidad, se fundamenta a partir de diferentes historias, las cuales narran sus experiencias personales, sus historias de vida a través de la apicultura, y cómo ésta dio paso a diferentes grupos para vincularse de cierta forma a una Política Pública, dando inicio a la institucionalización de la apicultura. En este apartado, Woortmann (1995) es usada para analizar desde la historia oral los procesos macro-históricos, estos son traídos y movilizados a partir de la memoria, entrelazándose en las historias de vida de las personas. Sin embargo, pensando en lo que la autora llamó mecanismos de resistencia cultural del campesino, son traídos a la discusión trabajos de Woortmann y Woortmann (1997) y Woortmann (2001) donde critican la perspectiva economicista de algunos autores sobre el campesino, ellos expresan por qué el "trabajar de la tierra" también debe ser visto como un valor ético, donde la tierra no es solo un factor de producción en donde se proyecta el trabajo, sino un valor moral. En ese sentido el campesino debe ser entendido como un orden moral y es a partir de su moralidad que el mundo del campesino se organiza, siendo el trabajo, el patrimonio de la familia, sobre el cual se construye la familia como valor (WOORTMANN, 2001).

En este apartado se evidencia el tránsito de lo local a lo regional, implicando la participación y presencia del Estado, lo que se puede pensar como "institucionalización" o formalización de la práctica, siendo el punto en que se evidencia lo que Pollak (2006) resalto de la historia oral como son las memorias subterráneas, "esas memorias subterráneas prosiguen su trabajo de subversión en el silencio y de manera casi imperceptible afloran en momentos de crisis a través de sobresaltos bruscos y exacerbados. La memoria entra en disputa" (POLLAK, 2006, p.18).

Por esta razón, el último apartado denominado La Asociación, si bien se muestra el espacio que ha ganado la apicultura en la vida de diferentes pobladores, dando paso a la articulación de varios grupos mediante la formación de la Asociación de Apicultores del Macizo ColombianoAPIMACIZO, también se hacen presentes los silencios sobre el pasado, los cuales no son más que razones políticas del silencio y esto se refleja al momento en que salen del olvido voluntario para ser revindicados en contextos específicos.

\section{Área de estudio y recolección de datos}

Colombia es un país caracterizado por una alta diversidad biológica donde se mezclan diferentes topografías con variedades de culturas, siendo ejemplo de ello la región denominada el Macizo Colombiano. Este es definido como un "complejo geohidrológico que cuenta con una alta biodiversidad y en conjunto, capta, almacena y regula el agua, que da lugar a los nacimientos de los ríos importantes en la vida y economía del país” (IDEAM, 1999, p.2). El Macizo Colombiano, según Gulh (1945), es un reflejo del sistema andino ecuatoriano de origen volcánico, cuyos depósitos de estos materiales llenaron y formaron cuencas que luego fueron atravesados por ríos; este borde del tronco andino desciende hacia la Amazonia colombiana, "exceptuando la parte fría, está cubierto totalmente por selvas húmedas, en las cuales se encuentran las cabeceras de las principales arterias fluviales de la Amazonia colombiana" (GULH, 1945, p.170).

Dentro de los municipios que integran el Macizo Colombiano se encuentra el municipio de La Vega, Cauca, el cual esta ubicado en la zona tórrida o tropical, caracterizándose por variedad de climas, que junto con sus diferentes pisos térmicos, permite el cultivo de gran variedad de productos agrícolas, lo cual ha sido históricamente relevante al hablar de sistemas agrarios en la región. De hecho, la agricultura desarrollada en esta región "presenta dos características: usan sólo mano de obra familiar y son autosostenibles. Los campesinos cultivan caña panelera, café, yuca y plátano, que se complementan con el pancoger" (MOLANO, 2011, p.2).

Es en esta región, específicamente en el año 2007, un grupo de familias campesinas productoras de miel y café tuvieron la iniciativa de comenzar un proceso de organización a partir de la práctica apícola. Así que, durante el 2008 este grupo decide pasar de ser un proyecto para convertirse en una Asociación, es finalmente en el 2009 cuando se crea jurídicamente la Asociación de Apicultores del Macizo Colombiano- APIMACIZO.

Figura 1: Localización geográfica del municipio de La Vega, Cauca 


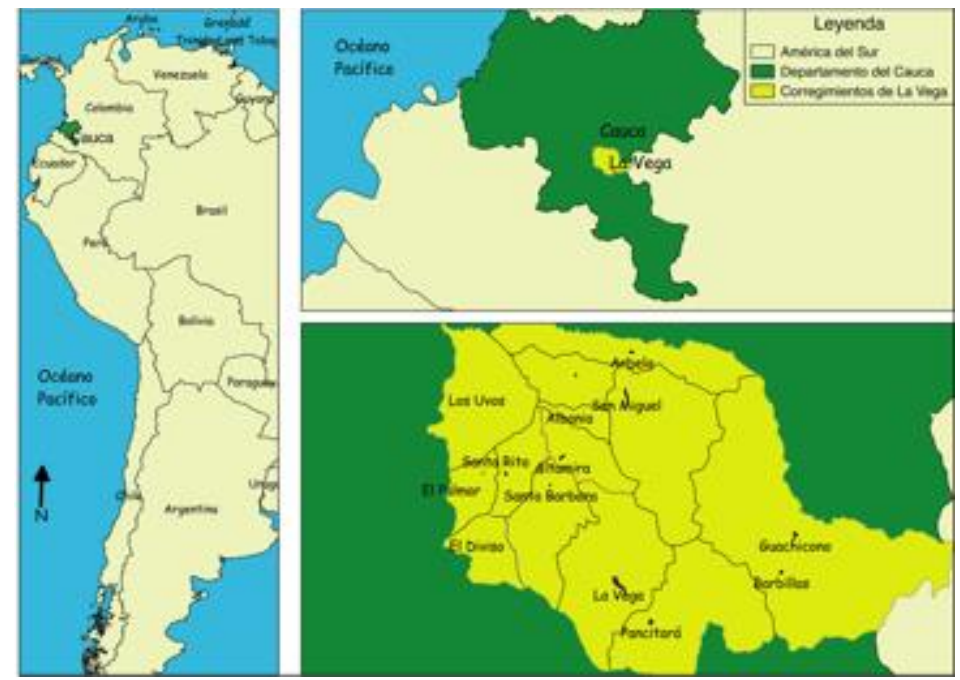

Fuente: Elaboración propia a partir do Sistema de Información Geográfica para la Planeación y Ordenamiento Territorial (Colombia) -SI-GOT-.

La pesquisa fue desarrollada entre el segundo semestre de 2016 y primer semestre de 2017. Sin embargo, es relevante aclarar sobre el conocimiento previo que se tenía de la Asociación y sus lideres. Partiendo de este hecho, fue realizado un sondeo previo a la implementación de la metodología historia oral, en donde fue posible caracterizar a las personas miembros de APIMACIZO que cuentan con experiencia de 20 y 40 años en la práctica apícola, quienes para efectos de esta pesquisa se les otorgó la denominación 'larga trayectoria'. Así que, al momento de implementar la historia oral fueron usados como recurso metodológico los relatos orales de los 11 miembros anteriormente caracterizados, del total de 41 registrados en esta pesquisa. Para efectos de esta pesquisa, fueron alterados y remplazados los nombres reales de los participantes por ficticios, buscando no comprometer la integridad de las personas entrevistadas.

Dentro del cuadro amplio de la metodología historia oral, fue usada historia de vida y memoria colectiva en el grupo de 11 apicultores. Historia de vida se puede definir como los relatos orales de un narrador sobre su existencia a través del tiempo intentando reconstruir los acontecimientos que vivió, en el que no se presenta diálogo entre el entrevistado y entrevistador o si se presenta es en lo más mínimo; es decir, quien dirige la conversación es el propio narrador (QUEIROZ, 1988). En el caso de memoria colectiva, retomamos autoras como Godoi (1999) y Woortmann (1995), para quienes los relatos nos remiten a un pasado general, lo cual va a constituir el primer dominio donde la memoria colectiva se cristaliza, se establece una distinción entre el tiempo pasado y el tiempo de hoy, marcando una división histórica fundamental. Esta a su vez, permite a un grupo no solo de definirse espacialmente, sino históricamente, siendo este último recreado según las condiciones sociales en las que están inmersos. Por lo tanto, el estudio de memorias como recurso metodológico del campo de la historia oral, posibilita el tránsito entre el pasado y el presente y trae a la luz, los cambios ocurridos a lo largo del tiempo (SILVA, 2008).

\section{Surgimiento y establecimiento de la apicultura en la región: un "nuevo" conocimiento en la producción}

Los sistemas de producción no son espacios vacíos, son espacios socialmente estructurados según las relaciones del saber (BRANDÃO, 1986). Para nuestro caso específico, en el trabajo con la apicultura, fue la iglesia como institución, quienes participaron en la introducción de esta nueva práctica en la región. Un saber que es documentado a partir de la memoria de sus actores. Los relatos proporcionados por estos apicultores nos remiten a la memoria colectiva de los miembros de APIMACIZO, destacando la participación de dos personas quienes fueron fundamentales para la apropiación y establecimiento de la práctica desde sus marcos de significación cultural (GONZÁLEZ, 2008).

A continuación, se presenta el relato de uno de los integrantes de APIMACIZO, en el año 2016, quien expone el contacto inicial de su familia con la apicultura como sistema productivo. 
de la iglesia, porque eso es grande, eran abejas italianas y como la abeja italiana no es agresiva, las tenía allá. Él tenía un apiario con varias colmenas, y mi papá y un tío fueron aprendiendo ahí. Antes de irse el Padre cuando lo trasladaron se las vendió a mi papá y le dejó explicando como era. Solo se producía miel, la cera sale por defecto, por la desoperculación, pero no era un negocio (Marcio, 46 años).

Como lo menciona nuestro interlocutor, su primer contacto con la apicultura es por parte del Padre, mediante el trabajo de su familia en colaboración a este. Es el Padre quien les da a conocer las comúnmente llamadas abejas italianas (Apis mellifera ligustica), siendo este recuerdo, así como el de otros miembros de la Asociación de 'larga trayectoria', el primer referente de la actividad apícola en el municipio. El Padre llegó a Colombia en el año 1955 y era diocesano, proveniente de Suiza, a quien se recuerda como una persona emblemática en la región, no solo por ser un representante de la iglesia, sino por su papel como "fundador" de la apicultura en el municipio. Sin embargo, según las personas de la región, al municipio llegó en 1960, enmarcándose su llegada a Colombia en el proceso de universalización de la iglesia católica. Estas misiones católicas que de Europa se dirigieron hacia Latinoamérica, "vinieron precisamente para colaborar en sitios donde había poca presencia de la iglesia [...] y venían con la intención de colaborar con proyectos de desarrollo en esa época" (Patricio, 80 años).

La principal función que el padre tenía en el municipio de La Vega era el fomento de la educación superior; aunque su participación en la zona no se limitó solo a esta actividad, de hecho, no es por ella que aparece en los relatos de nuestros interlocutores, sino, por el fomento de la apicultura que él y la iglesia toman relevancia: "fue una actividad que el padre conocía y él veía que eso para la gente podía ser, digamos, un apoyo económico y alimenticio. La intención de él era hacer conocer una cultura que podía servirle a la gente para mejorar la situación en el campo" (Patricio, 80 años)

El relato si bien podrá ser tomada como una memoria individual de nuestro interlocutor, como lo expresa Halbwachs, "cada memoria individual es un punto de vista sobre la memoria colectiva" (HALBWACHS, 2004, p.50). Los recuerdos siempre están asociados a un grupo, la reconstrucción de estos debe realizarse a partir de nociones comunes que se encuentran en la mente, nuestros pensamientos se originan en entornos y circunstancias sociales definidos (HALBAWCHS, 2004). De esta forma, y siguiendo el argumento analítico de Sanz (1998), esta memoria individual se transforma en colectiva en el momento en que es legitimada, compartida y preservada en el tiempo por los miembros de un grupo, siendo en este caso particular el relato del surgimiento de APIMACIZO. El recuerdo del Padre fue enfatizado por encima del recuerdo de otro sacerdote quien estuvo en la zona 10 años después de él, quien también colaboró con la práctica apícola, era suizo, y llegó en 1965 a Colombia, de quien afirman "llegó al municipio en 1970, se preocupaba mucho por la agricultura y ayudaba a los campesinos a modernizar y adaptarse en todo eso" (Patricio, 80 años).

Pero a diferencia del primero, son muy pocas personas quienes lo referencian, cuando lo hacen, es de forma escueta y fugaz al punto de no saber siquiera su procedencia.

En este sentido, como lo expone Brandão (1986), todas las comunidades deben ser entendidas como parte de formaciones inclusivas, en donde su propia realidad solo se da a través de relaciones de producción de bienes, servicios y con otros segmentos de la sociedad. En este estudio de caso, por lo tanto, la iglesia y su representación local en la figura del Padre es decisiva en la construcción de la memoria colectiva de los miembros de APIMACIZO. Estas son fuentes fiables de transmisión e información, que permiten una estructuración, reflejando los valores del grupo y el papel desempeñado por la institución, dándole estructura de legitimidad a la memoria y convirtiéndose en datos históricos insertados en ella (SANZ, 1998).

Al hacer referencia al momento del "surgimiento" de la apicultura en el municipio de La Vega, existe una figura representativa, como lo es el Padre. No obstante, en un segundo momento, durante el establecimiento de esta práctica aparece otra persona que sobresale en los relatos, como lo es el señor Cristán. Él es recordado como "el aprendiz" del Padre, quien una vez este último fue trasladado de diócesis, no solo le compró las abejas, sino que fue el encargado de continuar esta práctica en la región.

Yo le recomendé a don Cristán [...], que cuando fuera a cosechar miel, yo estaba listo, ahí en la finca. Yo solo en 2 ocasiones estuve con el Padre [...], él me recomendó que con don Cristán [...]yo podía participar con él, entonces 
don Cristán [...] cada que pasaba me echaba el grito y yo lo que estuviera haciendo lo dejaba y arrancaba, como era un poquito más abajo no más [la finca] y ahí empecé a ponerle mucho cuidado y a preguntar toda esa vaina, porque me interesaba mucho la apicultura (Melciades, 63 años)

Como se menciona en el parágrafo, nuestro interlocutor se encomendó al Sr. Cristán para poder aprender la apicultura, ya que, si bien había tenido contacto previo con el Padre en la práctica apícola, no había sido lo suficiente como para aprender dicha actividad. Siendo amigo y vecino del Sr. Cristán, al Sr. Melciades se le facilitó la transmisión del saber y fue a partir de la práctica y el leguaje oral, como él lo describe, que fue aprendiendo.

El Sr. Cristán junto con el Padre son recordados por su relación de amistad, la cual se entrelaza por el trabajo en la apicultura, en donde la innovación en esta práctica les permitió ganar un puesto en la memoria de las personas. La astucia de estas dos personas permitió incursionar en temas que para la década de los 70's con respecto a la apicultura no eran aún tratados en la región, como el caso de enfermedades que se presentaban durante el manejo de las abejas. La señora Antonia, esposa del Sr. Cristán, hace referencia de algunos métodos que inventaron el Padre y su esposo para tratar estas enfermedades. Por ejemplo, al mencionar la enfermedad del piojo de las abejas, la cual fue controlada por una técnica inventada por ellos, afirmaba que "eso se lo inventaron con el Padre", "eso sí se la llevaban con Cristán [...]”.

Por lo tanto, la memoria colectiva de nuestro grupo cumplió su función de seleccionar los hechos y protagonistas que para ellos influyen en la identidad de grupo, creando así un filtro en el cual los recuerdos seleccionados son los que fortalecen tanto el surgimiento de una práctica hasta el momento desconocida, y su posterior difusión a partir de dos actores sociales que hoy se configuran como los pioneros de la apicultura. Además, como lo menciona Brandão (1994), la memoria no solo son recuerdos y añoranzas, son sentimientos que van formando la identidad propia, en este caso particular, una identidad de apicultores que actualmente se consolida con la fundación de APIMACIZO.

\section{La localidad, grupos de apicultores en busca de complementariedad en la producción}

En el apartado anterior, la memoria colectiva de los campesinos agrupados en APIMACIZO revindicó la importancia y participación del Padre y el Sr. Cristán durante el surgimiento y consolidación de la apicultura en el municipio. Aunque, antes de la fundación de la Asociación, se crearon grupos pequeños que remiten a grupos domésticos o de vecinos, quienes empezaron a practicar la apicultura de manera no organizada a como está hoy en día. Así, una vez relatada la experiencia del surgimiento, la memoria pasa de ser colectiva a tornarse en experiencias personales de cada miembro de la Asociación entrevistado, los cuales hacen parte de recuerdos íntimos, de sensaciones propias y no colectivas, que vivieron durante el trabajo con la apicultura. Por lo tanto, con base en la metodología historia oral se analizaron las historias de vida de algunos miembros de APIMACIZO, recuerdos e imágenes personales que fueron vividas a través de la apicultura.

Las historias de vida obtenidas en diferentes espacios permitieron analizar algunas relaciones que se presentaron en el trabajo de la apicultura. Se evidenció que a partir del saber aprendido surgieron tres grupos en diferentes veredas del municipio, los cuales sirvieron de base para la conformación de lo que hoy es APIMACIZO. Con el objetivo de comprender cómo se dio este proceso de organización a manera de grupos domésticos y vecinales en torno a la actividad apícola, nos centramos en la relación de esta actividad con los saberes locales transmitidos de generación en generación, que serán descritos a continuación.

Yo tenia 10 años cuando comencé ayudarle a mi papá porque como [las abejas] no eran agresivas, eso fue mas o menos en el 80 , yo alcancé a trabajarle un tiempo antes de la africanización [de las abejas]; y más o menos en el 84-85 llegó la africanización, yo creo que llegaron del Brasil por el Amazonas, por acá abajo, por el Caquetá, no sé (...) La africanización aquí en la Vega llega en el 84-85, inclusive tuvimos que cambiar el equipo de manejo: ya tocó usar overol, ya tocó usar guantes largos, caretas más gruesas; porque antes simplemente se utilizaba una caretica en la nuca y sin guantes ni nada, eso era con la italiana (Marcio, hijo del Sr. Cristán). 
Este relato en el cual nuestro interlocutor reconstruye su historia a partir de algunos acontecimientos de su vida a través del tiempo, trae el recuerdo sobre cómo empezó a trabajar la apicultura, el cual nos coloca dos elementos importantes en la práctica apícola local: el primero, es el trabajo y aprendizaje familiar. El segundo, la temporalidad que está relacionada con el proceso de africanización de las abejas. Para comenzar, la familia es un elemento decisivo en la continuidad del campesinado, es a partir de su historia familiar como nuestro interlocutor se relaciona directamente con la producción apícola, siendo este un proceso de transmisión entre padre e hijo dentro del núcleo familiar. Es precisamente en la familia, donde nuestro interlocutor narra su colaboración en el proceso de aprendizaje y práctica misma de la apicultura, siendo la figura paterna quien cumple un papel jerárquico dentro de ésta. Partiendo de la experiencia del manejo apícola de su padre, el Sr. Marcio, logra aprender un sistema de producción, manteniéndose la relación padre e hijo. Por lo tanto, el carácter familiar es expresado en las prácticas sociales y esto implica una asociación entre el patrimonio, trabajo y consumo en el interior de la familia, lo cual es orientado a una lógica de funcionamiento especifico (Wanderley, 2003), organizándose en función de la experiencia en el trabajo, la cual implica jerarquía (WOORTMANN, 2001).

En el caso de nuestro interlocutor, la transmisión de la experiencia tiene su hilo conductor en el saber de la actividad apícola, la cual dentro de su familia comienza con la transmisión por parte del Padre, para después convertirse en un saber adaptado y direccionado por su papá. El paso de una generación a otra a partir del propio trabajo, los autores Woortmann y Woortmann (1997) lo definen como "saber-fazer", un saber que se gesta en la familia por parte de la figura paterna, el cual no es solo una transmisión de técnicas en la producción, sino que va a involucrar valores, construcción de papeles y jerarquías dentro del grupo familiar. Ese saber, por lo tanto, va a ser jerárquico y determinará el control sobre el grupo y en la familia donde se reproduce.

Por otra parte, el segundo elemento mencionado es el proceso de africanización de las abejas, un elemento que denota la temporalidad, expresa un tiempo específico en el que se pasó del uso de las abejas italianas (Apis mellifera ligústica) para las abejas africanizadas, en el trabajo apícola. El proceso mediante el cual se da el encuentro de estas especies de abejas, Martínez (2006) lo llamó el 'evento de 1983' para el caso Colombiano en general, es decir, evento donde se generaron híbridos entre los diferentes tipos de abejas que existían en el país para el uso apícola, como las abejas alemanas (Apis mellifera mellifera), italianas (Apis mellifera ligustica), caucasianas (Apis mellifera caucasica), africanas (Apis mellifera adansonii o scutellata), cuya denominación actual de africanizadas se debe a que presenta más características similares a las africanas (MANTILLA, 1997). Siendo este un proceso macro-histórico, en el sentido de que, así como para el Sr. Marcio implicó cambios en la forma de trabajar la apicultura, fue un proceso que se dio también a nivel nacional y en Latinoamérica.

La temporalidad dada en este relato, nos permite entender la adaptabilidad de este grupo familiar ante la nueva situación que enfrentaron al no desistir del trabajo apícola. Es de esta forma, como el Sr. Marcio y su papá, el Sr. Cristán, en la década de los 90 `s establecen un negocio familiar llamado Apiario el Macizo, en donde comercializaban miel en el municipio y en poblados cercanos, lo cual para este grupo familiar implicó la entrada de recursos y la consolidación de la apicultura como actividad económica. Aunque, para esta época aún no dimensionaban que este negocio se convertiría posteriormente en el punto de partida de un grupo más grande, del cual nos encargaremos de describir en el siguiente apartado.

La historia que presentamos a continuación es la representación del trabajo solidario en las labores agrícolas, que sin duda alguna representa la unión vecinal para disminuir las cargas de trabajar la tierra.

La historia de nosotros, de los abejeros se puede decir, que aquí hace unos 22 años que conformamos un grupo de trabajo, cambio de mano, entonces en los reposos de las comidas que teníamos nos poníamos hablar, éramos como 11 no más y nos poníamos hablar, y decíamos " hombre, aparte del plan de trabajo que teníamos de cambio de mano, pues hombre pensemos en hacer algotra cosa”, entonces y esa idea de las abejas la propuso Melciades [...], él estaba en el grupo de trabajo y cambio de mano, entonces le digo yo: "hombre pero que otra cosa puede ser que podemos coger fuera de esto". Melciades [...] dijo: "pues eso sí es fácil, podemos coger un trabajo de las abejas"; nosotros no sabíamos de eso nada y le dijimos: "bueno y ieso qué es?, ¿cómo es eso?". Melciades [...] dijo, "vea eso sí es fácil”. Nosotros le dijimos: “¿usted sabe?, 
¿tiene idea de eso?”; Melciades [...] dijo,: “yo tengo idea”, entonces le dijimos: “explíquenos y hagámosle” (Samiro, 67 años)

Este relato proporcionado por el Sr. Samiro, como él lo menciona, narra el comienzo de un grupo llamado ASPICOLAS, el cual en sus inicios era una forma de trabajo colectivo entre amigos y vecinos, forma de asociatividad en las labores agrícolas que es denominada localmente como "cambio de mano", y aunque puede variar su nombre dependiendo de la zona (jornal devuelto, a mano cambiada), tienen el mismo significado. Este tipo de trabajo es equiparable a lo descrito por Cândido (2010) al referirse al mutirão como trabajo colectivo vecinal, es una forma de solucionar el problema de mano de obra limitada con ayuda de un grupo de vecinos.

El grupo, según lo explicado por las personas que pertenecieron al mismo, se dedicaban a las deshierbas de las fincas, recolección de café en época de cosecha, entre otras labores, que demandaban mano de obra escasa en la región, o en su defecto, no se tenían los recursos económicos con qué pagarlos, tal y como lo menciona uno de nuestros interlocutores, el Sr. Aparicio (57 años): “ [el cambio de mano] nació de la necesidad de nosotros de los del grupo, porque pues no teníamos, económicamente estábamos mal y no podíamos pagar trabajadores, entonces yo te ayudo, tu me ayudas y así sucesivamente”.

Los integrantes del grupo trazaban un plan de trabajo diario. Cada día era destinado para al trabajo en la finca de uno de sus miembros, a donde todos se desplazaban y realizaban la actividad acordada. Entre esta rutina y los intervalos de descanso, las reflexiones nunca faltan, surgieron preguntas sobre, qué otras actividades realizar, cómo lograr complementar sus labores diarias con alguna otra que generara más que nuevos ingresos económicos a los grupos domésticos, alguna complementariedad alimenticia, siendo la apicultura una de las soluciones propuesta por el Sr. Melciades, quien como recordamos durante el establecimiento de la apicultura, también había conocido al Padre y tenía nociones básicas sobre esta práctica con el Sr. Cristán. De esta forma y siguiendo la sugerencia y conocimientos previos del Sr. Melciades, el grupo accedió a empezar a trabajar la apicultura a principios de la década de 1990.

la idea que yo les decía era mantener 1 o 2 colmenitas por participante para tener un complemento alimenticio en la casa, porque en ese tiempo para conseguir una media de leche tocaba que torear un sapo, entonces les dije "la miel tiene tantas propiedades, que supera la leche, supera la carne, echándoles toda esa vaina, y como es cierto [...] entonces lo miramos así, como un complemento alimentario en la familia (Sr. Melciades).

Es de esta forma, como el grupo vio en la apicultura un complemento que con el tiempo no solo era alimenticio, sino que fue tornándose un complemento económico, tanto de la caficultura, como de los otros cultivos que tenían. Después de haber tenido éxito en la comercialización de miel, resolvieron legalizarse jurídicamente, así que tramitaron la personería jurídica de su grupo en la Cámara de Comercio del Cauca, lo que implicó la legalización comercial de ASPICOLAS.

A partir de esta forma organizativa, lograron acceder a unos recursos por parte del Comité Departamental de Cafeteros, así como lo expresa el Sr. Melciades: "el Comité de Cafeteros nos dio mucho la mano, nos consiguió unos recursos [...], nos consiguió un millón de pesos [valor aproximado en dólares en el año 1990 sería de US\$ 1968]”, recursos que fueron usados para mejorar la infraestructura de los apiarios, logrando dotarse de instrumentos que requerían prioritariamente, como es el caso de la centrifuga de miel. Este equipo era fundamental para extraer la miel, ya que la manera en que realizaban esta actividad era manera manual, lo que les ocasionaba problemas y dolores en las manos.

Por lo tanto, desde el primer paso que dieron para institucionalizar su práctica, empezaron a participar en procesos de capacitación con el objetivo de ir tecnificando gradualmente la actividad apícola, pero también de ampliar la comercialización de miel; es en este proceso que fue pasando el tiempo y llegaría el momento en que los esfuerzos y el trabajo en equipo daría cabida a un nuevo proceso de agrupamiento que será explicado en el apartado denominado "Asociación".

En un último relato antes de dar paso al momento de la unificación en donde se crea APIMACIZO, nos remitimos a otro grupo de apicultores creado en la Vereda La Betulia, que fue denominado ASDECOB -Asociación de Desarrollo Comunitario La Betulia, la cual también empezó como una iniciativa grupal de varios vecinos, pero al final quedó integrada únicamente por dos personas. Al respecto, el Sr. Augusto nos comenta que: 
Trabajamos harto tiempo con Cristán, harto tiempo, hasta que yo hice una organización de ahí de la vereda, con unos amigos que trabajamos, hice un proyectico y el Comité de Cafeteros nos hizo una ayuda, compramos algunos materiales, pero los compañeros ninguno les gustó, nos quedamos dos. (Sr. Augusto, 73 años)

El Sr. Augusto, como lo menciona en la cita, trabajó por mucho tiempo con el Sr. Cristán en "sociedad". Sin embargo, decide separarse de don Cristán para formar su Asociación junto con otros vecinos en la década de 1990, pero debido al rechazo de sus vecinos a esta práctica "nueva", quedó solo en compañía del Sr. Hermes. Ellos continuaron trabajando, aunque como el anterior grupo, nunca pensaron en la apicultura como un negocio, sino que fue pensada también en el autoconsumo, o para regalar a los amigos.

En aquella época la miel era el único producto que conseguían cosechar, pero en la región no se conocía los beneficios de este producto, como lo menciona nuestro interlocutor: "la gente no tenía la cultura de la miel, solo los médicos yerbateros [médicos naturales o tradicionales], que le decían a uno échele a esta agüita dos góticas de miel, de resto esa cultura del consumo de la miel no se tenia" (Sr. Augusto). Así que, aunque recibieron la ayuda del Comité de Cafeteros en ese momento, nunca pensaron en un gran negocio, su iniciativa en la apicultura fue pensada en la diversificación en la producción familiar, es solo a partir de la formación de APIMACIZO que ellos le encontraron una posibilidad de ganancia en la práctica que ya venían realizando.

\section{La asociación: la unión entorno de APIMACIZO}

La asociación es el nombre que fue dispuesto para esta sección final, ya que es el momento en donde se presenta el punto de encuentro de diferentes personas y sus respectivas historias, las cuales fueron relatadas desde una noción de grupo y también desde las vicisitudes de cada vida que participó en esta pesquisa; relatos que permitieron entender las relaciones sociales que se tejen alrededor de una práctica, como lo es para nuestro caso la apicultura.

Como se mencionó en el inicio del texto, la dinámica de la memoria de nuestro grupo nos llevó a recorrer la historia compartida del inicio de la apicultura. Sin embargo, como menciona Jelin, "la memoria como construcción social narrativa implica [...] también prestar atención a los procesos de construcción del reconocimiento legítimo, otorgado socialmente por el grupo al cual se dirige" (JELIN, 2002, p. 35). Por eso, entre las historias personales, nociones familiares y vecinales, dieron cabida a diferentes grupos de la zona, donde finalmente surge la creación de APIMACIZO. En este punto, aparecen tensiones y contradicciones a partir de varios acontecimientos e incorporación en la región de algunas políticas de gobierno de la época que hacen posible dicha unificación, sobre lo cual trataremos a continuación.

Lo que ocurre es que cuando llegó Guardabosques, había que ahorrar, una de las condiciones de guardabosques era el ahorro voluntario, que no era ni tan voluntario, pero era un ahorro, y se podía usar ese ahorro en un proyecto productivo, obviamente en el caso mío opté por la apicultura, adicionalmente a eso, cuando guardabosques se creó, las cooperativas, la nuestra, la que yo gerenciaba, porque yo fui el gerente de VEGACOOP, se llamaba Cooperativa Multiactiva Villa Real de la Vega, entonces el ahorro había que usarse en lo productivo, y utilicé yo en mi caso y otros 17 guardabosque para eso (Marcio, 46 años).

El programa de Familias guardabosque (PFGB) que esta nombrando nuestro interlocutor hizo parte de las "políticas públicas de lucha antinarcóticos y defensa nacional" (CASTAÑO, 2016, p.183); era una estrategia que buscaba fomentar la "cultura de legalidad" en población con riesgo o afectada por cultivos ilícitos en el sector rural. Esta se implementaba mediante "un compromiso de no siembre ni resiembra de cultivos ilícitos [...]entrega a cada familia un incentivo económico" (UNODC, 2009, p.9).

El PFGB estaba inscrito dentro de la continuidad y mejoramiento del "Plan Colombia" y la "Política de Defensa y Seguridad Democrática" para el periodo de gobierno nacional comprendido entre 2002-2006, siendo el 2004 el año en que fue beneficiada la población de la Vega, Cauca. El programa de familias guardabosques si bien se enfocó en el desarrollo alternativo en las zonas 
rurales, nunca fue pensada como una Política Pública de desarrollo rural, estuvo pensada más a favor de las estrategias de "erradicación de cultivos ilícitos voluntaria" en zonas donde estos existían o se presumía que existían. Nuestro interlocutor hizo parte de este programa, así como 17 personas más, según los miembros de APIMACIZO se les llama "los guardabosques". Ellos debían ahorrar una parte del dinero que fue dado por el programa para la sustitución de cultivos, teniendo como finalidad la inversión en un proyecto productivo, por lo que la propuesta del Sr. Marcio para los otros 17 guardabosques fue trabajar la apicultura.

La propuesta de trabajar con apicultura ganó adeptos a causa de dos razones: la primera, muy señalada en el municipio, es la poca demanda de extensión de tierra si se compara con otras actividades productivas, tal y como lo menciona el Sr. Augusto (73 años): "pa ganadería se requiere extensión de tierras, para el café pues por la altura no; es alto en La Vega [específicamente en la zona "Alta" del municipio] entonces no le funciona, y la apicultura no necesita una extensión de tierra para instalar el apiario". La segunda razón fue la muy nombrada y conocida experiencia que tenia el Sr. Marcio y su papá en el manejo apícola, como lo vimos anteriormente, quienes ya desde la década de 1990 venían produciendo y comercializando miel bajo el nombre de Apiario el Macizo.

Este pequeño grupo de 17 campesinos locales quienes estaban asociados en la Cooperativa Multiactiva Villa Real de la Vega-VEGACOOP en ese momento, decidieron implementar los recursos del programa de guardabosques en un proyecto de producción y comercialización de miel a un nivel municipal, motivo que los llevó a tomar la decisión de ampliar el grupo y, con ello, emprender la búsqueda de otras personas de La Vega que ya tuvieran alguna experiencia en esta práctica y estuvieran interesadas en acompañarlos en dicho proyecto. "Entonces los 17 guardabosques salimos a buscar a las personas [...], que tenían 2 o 3 colmenitas, entonces hicimos las visitas, los georreferenciamos [...] de esos entraron 22 [personas más]" (Sr. Marcio, 46 años).

Es en este punto, en donde los relatos adquieren silencios colectivos, se habla de hacer parte del programa familias guardabosques, pero nunca se habla del por qué ingresaron al programa, en qué punto ellos y sus familias fueron participes de lo que el Estado llamó "cultura de la ilegalidad". Las historias de vida, en el sentido de Pollak (2006), son al igual que la memoria colectiva, susceptibles a ser presentadas en función del contexto en que son relatadas. Siendo la historia de vida considera por este autor un "instrumento de reconstrucción de identidad y no solamente como relatos factuales" (POLLAK, 2006, p.30). Así que, como cualquier proceso discursivo, se generan un sinnúmero de contradicciones y tensiones, siendo el silencio una forma de acomodación al medio social. Según Rios (2013), el silencio debe ser entendido como una condición necesaria de sobrevivencia para los recuerdos de los grupos "subyugados".

Si bien se presentaron silencios, esas memorias subterráneas sufren un proceso de activación bajo contextos institucionales, al querer ser revindicadas en espacios donde los procesos de eliminación y cambio de cultivos ilícitos pueden implicar una reafirmación de su identidad. En ese sentido, Jelin afirma que "no hay un único tipo de olvido, sino multiplicidad de situaciones en las cuales se manifiestan olvidos y silencios con diversos "usos y sentidos"”, porque "el pasado que se rememora y se olvida es activado en un presente y en función de expectativas futuras" (JELIN, 2002, p.29).

De las 22 personas nuevas que se adhirieron a la propuesta, de lo que posteriormente se llamaría APIMACIZO, ninguno hacia parte de familias guardabosques. Según lo expresado en algunas historias orales, las veredas en que dicho programa se intentó implementar, se opusieron hacer parte de este. Debido al descontento que causaba para algunas familias ser llamadas de cultivadoras de ilícitos, ocasionando que en varias veredas se organizaran para oponerse rotundamente, argumentando que ellos no cultivaban nada ilegal.

De estas 22 personas, algunas hacían parte de grupos ya conformados como ASPICOLAS quienes con el tiempo habían logrado tener una mejora en la práctica apícola y tener un mercado a nivel local. Otros hacían parte de ASDECOB, quienes habían continuado trabajando la apicultura en sus fincas, una apicultura pensada en lo familiar y se podría decir que hasta vecinal, los demás eran personas del municipio que ya venían de un trabajo previo, pero llevaban el trabajo de la apicultura de manera individual. Sin embargo, cada una de estas personas que ingresaron al proyecto, tenían alguna persona conocida dentro del grupo de "los guardabosques", y más importante aún, en su mayoría en determinado momento de sus vidas habían conocido la apicultura con el Sr. Cristán o, para el caso de los más jóvenes, con el Sr. Marcio. En otras palabras, la apicultura en el municipio de La Vega, por decirlo de alguna forma, tenía la imprenta de la familia de Cristán y de su hijo, el Sr. Marcio. 
Para el año 2007 en que se conforma este grupo de 39 personas, como bien se dijo, estaban aún asociados en la cooperativa VEGACOOP y no en APIMACIZO, ya que este era solo el nombre del proyecto que los había unido. Una vez la cooperativa no estaba siendo funcional para las metas y perspectivas que ellos se estaban proponiendo, deciden conformar la nueva asociación. Así, como manifiesta uno de los integrantes más jóvenes de la actual Asociación, "en el 2007 nace el grupo, en el 2008 se le da en nombre de Asociación, y en el 2009 se le da la personería jurídica" (Airón, 33 años).

Tomada la decisión de crear una nueva figura jurídica, en el año 2009 legalizan su organización mediante la figura de personería jurídica como la Asociación de Apicultores del Macizo Colombiano-APIMACIZO, a partir de allí, han logrado participar en la convocatoria de varios proyectos productivos como ADAM- Programa de Áreas de Desarrollo Alternativo Municipal, Proyecto Apoyo a Alianzas Productivas - PAAP, el cual es un instrumento del Ministerio de Agricultura y Desarrollo Rural que vincula a pequeños productores rurales con los mercados a través de un esquema de agronegocio con un aliado comercial formal, entre otros.

De esta forma, APIMACIZO ha logrado establecer puntos de comercialización en diferentes supermercados de la ciudad de Popayán, capital del departamento del Cauca, posicionándose con su marca "APIMACIZO, 100\% natural", no solo comercializando miel, sino no también una mezcla de "Propóleo en miel" el cual tiene un uso terapéutico para problemas respiratorios.

\section{Conclusiones}

Las narraciones proporcionadas por algunos de los miembros de APIMACIZO permitió entender la articulación de diferentes sectores (iglesia, familia, vecinos) en la vida del campesinado de esta asociación con la práctica apícola; donde la articulación se dio por medio del saber. Es a partir del saber en la apicultura, donde se consolidaron los lazos de amistad, vecindad, compadrazgo y las relaciones familiares, las cuales se reflejan al momento del trabajo colectivo en las diferentes labores agrícolas, demostrando que la producción marcará de modo decisivo las formas de organizar la vida social de los miembros de APIMACIZO.

A partir de las historias orales proporcionados por los interlocutores, sobresalen dos momentos clave en la configuración de la cuestión agraria de Colombia. El primero es en la década de los 90`s, el surgimiento de un proceso de asociatividad y colectivización. El segundo, es la implementación del programa familias guardabosques, donde ingresa el elemento de "cultivos ilegales" dentro de la población, siendo este ultimo el programa que direccionó y vinculó a APIMACIZO al acceso a proyectos de fomento nacional, evidenciando un nuevo enfoque sobre la ruralidad del país.

Por otro lado, la memoria colectiva presentó una fase de silencios voluntarios colectivos en los miembros de la asociación, lo cual se evidenció al momento de indagar sobre el contenido de familias guardabosques. De esta forma, la reacción de los entrevistados permitió comprender que muchas veces hay recuerdos que por voluntad propia un grupo desea omitir y eso es traducido en silencios u olvidos voluntarios.

Pensando en los sentimientos que moviliza la memoria, las contradicciones y tensiones que pueden llegar a aflorar las diversas reacciones de los entrevistados, nos permite cuestionarnos, en qué punto estas tensiones implicaron construir esa identidad que reivindica al campesino con una fuerte relación con la naturaleza, y cómo la historia se conecta con las experiencias personales en donde pueden existir zonas de sombras silenciosas.

\section{Agradecimientos}

Las autoras agradecen a CAPES -Coordenação de Aperfeiçoamento de Pessoal de Nível Superior, por la bolsa recibida para desarrollar esta pesquisa.

\section{Referencias}

BRANDÃO, Carlos. Saber e ensinar: três estudos de educação popular. $2^{\mathrm{a}}$ ed. Campinas: Papirus, 1986.

BRANDÃO, Carlos. O sentimento do mundo: memória, destino e cenários de vida entre errantes mineiros. In: A. Meneses, et al (Org.). As Faces da memória. Campinas: UNICAMP. 1994. p.61-84. 
CÂNDIDO, Antonio. Os parceiros do Rio Bonito: estudo sobre o caipira paulista e a transformação dos seus meios de vida. 11. ed. Rio de Janeiro: Ouro sobre azul, 2010.

CASTAÑO, Hernán D. Vélez. El programa Familias Guardabosques: una mirada al discurso político del Gobierno de Álvaro Uribe Vélez. Revista Trabajo Social, (18), 179-197, 2016.

DANE-Departamento Administrativo Nacional Estadística. (2014). Censo Nacional Agropecuario 2014. Recuperado de <https:/www.dane.gov.co/files/images/foros/foro-de-entrega-de-resultadosy-cierre-3-censo-nacional-agropecuario/CNATomo2-Resultados.pdf $>$ Acesso em 03/ 06/17.

FALS BORDA, Orlando. Retorno a la tierra (Historia doble de la Costa 4). Bogotá: Universidad Nacional de Colombia-Banco de la República-El Áncora Editores, 2002.

FORERO, J., GARAY, L. J.; BARBERI, F., RAMÍREZ, C., SUÁREZ, D. M. y GÓMEZ, R. Reflexiones sobre la ruralidad y el territorio en Colombia. Problemáticas y retos actuales. En: La eficiencia económica de los grandes, medianos y pequeños productores agrícolas colombianos. Garay, L.J. et al. Bogotá: Oxfam, 2013. p. 69-113.

GODOI, Emilia P. 0 trabalho da memória: cotidiano e historia no sertão do Piauí. Campinas: Editora da Unicamp, 1999.

GONZÁLEZ, María Vargínia. Agroecología: saberes campesinos y agricultura como forma de vida. México: Universidad Autónoma de Chapingo, 2008.

GUHL, Ernesto. El Macizo Colombiano: Informe preliminar sobre un ensayo etnográfico. Boletín de Arqueología, 1 (3), 257-265, 1945.

HALBWACHS, Maurice. La mémoire collective. [La memoria colectiva](1 ${ }^{\mathrm{a}}$ ed.). Zaragoza: Prensas Universitarias de Zaragoza, 2004.

IDEAM-Instituto de Hidrología, Meteorología y Estudios Ambientales. El Macizo Colombiano y su área de influencia inmediata: diagnóstico, descripción de la unidad regional y propuesta de delimitación (V.1).Santa fe de Bogotá, Colombia, 1999.

JELIN, Elizabeth. Los trabajos de la memoria. España: Editora Siglo XXI, 2002.

MANTILLA, Constantino. Principios de apicultura africanizada. Medellín: Editorial Universidad Nacional de Colombia, 1997.

MARTÍNEZ, Telmo. Diagnostico de la actividad apícola y de la crianza de abejas en Colombia. Bogotá: Ministerio de Agricultura y Desarrollo Rural \& Instituto Interamericano de Cooperación para la Agricultura (IICA), 2006.

MINISTERIO DE AGRICULTURA Y DESARROLLO RURAL DE COLOMBIA. Proyecto apoyo a alianzas productivas: apertura del registro de perfiles de alianzas 2016-2018. (2016). Recuperado de

<https://www.minagricultura.gov.co/convocatorias/Documents/Apertura_Registro_2016_2018/TdR _Alianzas_Productivas_2016.pdf $>$. Acesso em 28/07/17.

MOLANO, Alfredo. Dignidad campesina: entre la realidad y la esperanza. Bogotá, Colombia: ICONO Editorial, 2013.

PNUD- Programa de Naciones Unidas. Colombia rural. Razones para la esperanza: Informe Nacional de Desarrollo Humano 2011. Colombia: Bogotá, 2011. 
POLLAK, Michael. Memoria, Olvido, Silencio. La producción social de identidades frente a situaciones límite. La Plata, Argentina: Ediciones Al Margen, 2006.

QUEIROZ, Maria Isaura P. Relatos rurais: do "indizível ao "dizível”. En: O. Von Simson (Org.). Experimentos com história de vida (Itália-Brasil). São Paulo: Vértice, 1988., p.14-43.

RIOS, Fábio. Memória coletiva e lembranças individuais a partir das perspectivas de Halbwachs, Michael Pollak e Breatriz Sarlo. Revista Intratextos, 5(1), p. 1-22, 2013.

SANZ, María Alexia. Los recuerdos, la memoria colectiva y la historia, o como un pueblo construye su ayer. Anales de la fundación Joaquín Costa, (15), p. 217-238, 1998.

SILVA, Maria Andreia de Paula. Greve na Fazenda. En: D. Neves y M. Silva (Orgs). Processos de constituição e reprodução do campesinato no Brasil Formas tuteladas de condição camponesa. Brasil: Editora UNESP, 2008, vol. 1, p. 207-232.

UNODC- Oficina de las Naciones Unidas contra la Droga y el Delito.(2010). Des-Cifrando Las Familias Guardabosques: Evaluación Final Consolidado Nacional Línea Base, Seguimiento y Línea Final 2010. Recuperado de $<$ https://www.unodc.org/documents/colombia/2013/Agosto/DA2013/Descifrando_Fases_I_II_Linea Final.pdf $>$ Acesso em 28/07/17.

WANDERLEY, Maria Nazareth B. Agricultura familiar e campesinato: rupturas e continuidades. Estudos Sociedade e Agricultura. Rio de Janeiro: CPDA/MAUAD, (21), p. 42-46, 2003.

WANDERLEY, Maria Nazareth B. A sociologia rural na américa latina: produção de conhecimento e compromisso com a sociedade. ALASRU 2010. Asociación Latinoamericana de Sociología Rural, Porto de Galinhas, Brasil. Recuperado de http://www.alasru.org/wpcontent/uploads/2011/06/Nazareth-Wanderlei.pdf

WANDERLEY, Maria Nazareth B. O campesinato brasileiro: uma história de resistência. RESR, Piracicaba- SP, 52(1), S025-S044, 2014.

Woortmann, Ellen. Herdeiros, Parentes e Compadres: colonos do Sul e sitiantes do Nordeste. Brasília: UNB, 1995

WOORTMANN, Ellen e WOORTMANN, Klaas. O trabalho da terra: a lógica e a simbólica da lavoura camponesa. Brasília: Editora Universidade de Brasília, 1997.

WOORTMANN, Klass. O modo de produção doméstico em duas perspectivas: Chayanov e Sahlins. Brasília: Série Antropologia, UNB, 2001. 